\title{
Cavity Solitons in Two-Level Lasers with Dense Amplifying Medium
}

\author{
V. Ahufinger, ${ }^{1}$ J. García-Ojalvo, ${ }^{2,3}$ J. Mompart, ${ }^{1}$ M. C. Torrent, ${ }^{2}$ R. Corbalán, ${ }^{1}$ and R. Vilaseca ${ }^{2}$ \\ ${ }^{1}$ Departament de Física, Universitat Autònoma de Barcelona, E-08193 Bellaterra, Spain \\ ${ }^{2}$ Departament de Física i Enginyeria Nuclear, Universitat Politècnica de Catalunya, Colom 11, E-08222 Terrassa, Spain \\ ${ }^{3}$ Center for Applied Mathematics, Cornell University, Ithaca, New York 14853, USA
}

(Received 4 February 2003; published 22 August 2003)

\begin{abstract}
Local-field effects are known to induce bistability in dense optical media. We examine theoretically whether this property is preserved in broad-area cavities, and show that bistability between the homogeneous lasing and nonlasing states of the system persists provided a Fourier filtering technique is used to prevent off-axis emission. The resulting bistability gives rise to spatial light localization in the form of cavity solitons, which exhibit a particularly large degree of plasticity as a function of the characteristics of the addressing beam. This is the simplest laser able to sustain cavity solitons.
\end{abstract}

DOI: 10.1103/PhysRevLett.91.083901

Spatial solitons are nonspreading light beams which propagate through certain nonlinear media while keeping their transverse cross section unperturbed, due to a precise compensation of the naturally occurring beam diffraction with material-induced self-focusing [1]. These structures have potential technological applications in communications and information processing [2]. Besides propagation, a natural interest lies in the generation and storage of these structures. To that end, recent efforts have been addressed to confine spatial solitons within optical cavities. Cavity solitons have been predicted [3] and observed experimentally [4] in a large variety of passive (i.e., externally driven) optical media. Undoubtedly, it would be highly desirable to generate localized structures in amplifying cavities (i.e., in lasers). However, so far laser cavity solitons have only been shown to exist provided a passive element (a saturable absorber) is placed within the cavity [5] or in the presence of two-photon amplification [6]. In the present Letter, we show that cavity solitons can also be generated in simple, purely active two-level lasers, provided the amplifying medium is dense enough to make dipoledipole interactions important, which gives rise to localfield effects [7].

The influence of local-field effects on the propagation of coherent light beams through two-level systems has been substantially studied in the past. In particular, localfield corrections have been shown to give rise to a redshift in the resonance line of the medium (known as Lorentz shift) [8], and to produce intrinsic optical bistability [9], effects which have both been verified experimentally $[10,11]$. In this Letter, we study the effect of these corrections in an amplifying medium placed inside an optical cavity; i.e., the coherent field is generated by the atomic medium instead of being applied from the outside. Recent studies have analyzed the influence of local-field effects on temporal laser instabilities [12]. Here we consider the spatiotemporal case of a broad-area cavity, which will be shown to lead to the formation of cavity solitons, provided an appropriate spatial filtering is used
PACS numbers: 42.65.Tg, 05.45.Yv, 42.50.Hz, 42.65.Sf

to prevent destabilization of the nonlasing state and maintain bistability. An important peculiarity of the resulting solitons is their high level of plasticity, which allows a substantial control of the strength and shape of the soliton by adjusting the properties (peak intensity and width) of the addressing beam.

The interaction between a coherent beam and a dense two-level medium can be described in a semiclassical framework by a set of Maxwell-Bloch equations, which in the rotating wave and slowly varying envelope approximations can be written in the dimensionless form:

$$
\begin{aligned}
\dot{\alpha} & =-\sigma \alpha+i p \rho_{01}+i a \nabla_{\perp}^{2} \alpha, \\
\dot{d} & =b(r-d)-4 \operatorname{Im}\left(\alpha \rho_{01}^{*}\right), \\
\dot{\rho}_{01} & =-\left[1+i\left(\Delta_{c}+L d\right)\right] \rho_{01}-i \alpha d,
\end{aligned}
$$

where $2 \alpha$ is the Rabi frequency of the generated field, $\rho_{01}$ is the atomic coherence between levels $|1\rangle$ (ground state) and $|0\rangle$ (excited level), which is directly related to the polarization of the medium [13], and $d$ is the population inversion of the transition. All field amplitudes and decay rates are expressed in units of the relaxation rate $\gamma_{\perp}$ of the coherence $\rho_{01}$, whereas time is expressed in units of $\gamma_{\perp}^{-1} . \Delta_{c}=\left(\omega_{01}-\omega_{c}\right) / \gamma_{\perp}$ denotes the detuning between the transition frequency and the cavity resonance frequency. The transverse Laplacian $\nabla_{\perp}^{2}$ in Eq. (1a) accounts for light diffraction in the plane transverse to propagation. The diffraction coefficient is $a=c^{2} /\left(2 \omega_{c} w_{0}^{2} \gamma_{\perp}\right)$, where $c$ is the speed of light and $w_{0}$ is the beam waist [14]. The cavity decay rate $\sigma$, the pump rate $r$, and the decay rate of the population inversion $b$ are considered to be uniform in the transverse plane.

In high-density media, the microscopic electric field acting locally on the material differs from the macroscopic field. In the framework of the semiclassical laser theory, this difference leads to a nonlinear shift in the frequency of the atomic transition, which originates the local-field correction (LFC) term added to Eq. (1c). This nonlinear detuning, $\Delta^{s}=L d$, is the Lorentz shift. The LFC parameter has the form $L=p \gamma_{\perp} / 3 \omega_{c}$, where 
$p=\Pi / \Pi_{0}$ is the ratio between the density of the active medium $\Pi$ and a reference value $\Pi_{0}$, which is the one included in the normalized variables via the gain parameter [15]. We note that we have chosen a normalization different from the standard one [12] in order to make the dependence of the LFC parameter on the atomic density explicit.

In the case of a weak probe field the population difference, and hence the resonance-line shift, is practically constant for all detunings. This is the well known case of the static Lorentz shift. When the coupling field is not applied from the outside but is generated by the active medium, the population difference depends on the generated intensity: the highest intensity corresponds to the lowest population difference, i.e., the population difference at threshold. This population distribution gives rise to the dynamic Lorentz shift shown in Fig. 1(a), which represents the steady-state intensity versus cavity detuning for two different values of the local-field correction $L$ in the absence of transverse effects $(a=0)$. With a fixed $p, L$ can be varied by changing $\gamma_{\perp}$ and/or $\omega_{c}$. Note that for large values of $L$ the shift of the peak intensity is rather small, but the displacement of the basis of the line (where the population inversion is maximum) is very large. Such large $L$ can be obtained experimentally, for instance, in gas lasers operating in the far-infrared.

The steady-state solutions represented in Fig. 1(a) can be obtained from the Maxwell-Bloch equations (1) with $a=0$, which leads to an implicit equation for the imaginary part $y_{01}$ of the atomic coherence: $0=$ $y_{01}\left[A+B y_{01}^{2}+C y_{01}^{4}\right]$, where $A=(1+\sigma)^{2}(\sigma-p r)+$ $\sigma\left(\Delta_{c}+L r\right)^{2}, \quad B=4 b p\left[-2 L \sigma\left(\Delta_{c}+L r\right)+p(1+\sigma)^{2}\right]$, and $C=16 L^{2} p^{2}$. This equation has a trivial solution, $y_{01}=0$, corresponding to the nonlasing state $\alpha^{\mathrm{tr}}=0$, $\rho_{01}^{\mathrm{tr}}=0, d^{\mathrm{tr}}=r$. The biquadratic equation has at most four mathematical solutions $\left(y_{01}= \pm \sqrt{t_{1}}, \pm \sqrt{t_{2}}\right)$, representing only two different physical states (due to the
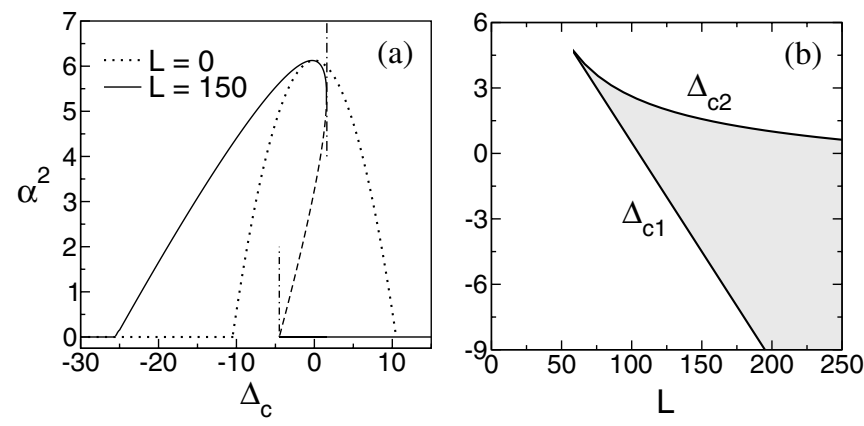

FIG. 1. (a) Intensity of the generated field as a function of the cavity detuning for $p=250, \sigma=0.5, b=0.5, r=0.1, a=0$, and different values of the LFC parameter: $L=0$ (dotted line) and 150 (solid line for the stable part, dashed line for the unstable one). The vertical dot-dashed lines indicate the limits of the bistability region for the last case. (b) Corresponding limits $\Delta_{c 1}$ and $\Delta_{c 2}$ of the bistability region (grey area) as a function of the LFC parameter. invariance of the model under a sign change of the coherent terms). When two different and positive values $t_{1}$ and $t_{2}$ exist, the system exhibits three steady-state solutions. The cavity detuning range in which these three solutions appear is given by $\Delta_{c 1}<\Delta_{c}<\Delta_{c 2}$, where

$$
\begin{aligned}
& \Delta_{c 1} \equiv-L r+(1+\sigma) \sqrt{\frac{p r}{\sigma}-1}, \\
& \Delta_{c 2} \equiv \frac{p(1+\sigma)^{2}}{4 L \sigma}-\frac{L \sigma}{p} .
\end{aligned}
$$

Note that only $\Delta_{c 1}$ depends on the pumping rate $r$, and that for a fixed value of the local-field correction there is always a threshold value of $r$ above which the bistability region appears. In this region, the lasing solution with the highest intensity and the nonlasing solution are stable, while the other lasing solution is always unstable [12]. Therefore, the region given above corresponds to a $b i$ stability region. For the parameters of Fig. 1(a) with $L=$ 150 , the limits of that region are given by $\Delta_{c 1}=-4.5$ and $\Delta_{c 2}=1.6$. Figure 1(b) shows the limits of the bistability region as a function of the LFC parameter $L$, for a fixed value of the gain parameter. One can see that there is a minimum value $L_{\text {th }}$ above which bistability occurs, which can be easily obtained imposing $\Delta_{c 1}=\Delta_{c 2}$ in (2), leading to $L_{\text {th }}=p(1+\sigma) / \sqrt{4 \sigma(p r-\sigma)}$.

In the presence of transverse diffraction, the system can develop a lasing solution with a small wave vector component transverse to the cavity axis [16]. Therefore, let us consider now the stability of the nonlasing solution versus a perturbation in the form of a transverse traveling wave $\left(\alpha=\delta \alpha e^{i(\vec{k} \cdot \vec{x}-\omega t)}, \rho_{01}=\delta \rho e^{i(\vec{k} \cdot \vec{x}-\omega t)}, \quad d=\delta d\right)$, where the wave vector $\vec{k}$ and the position vector $\vec{x}$ are perpendicular to the laser cavity axis. A linear stability analysis of the nonlasing solution in front of perturbations of this type indicates that, for a given value of $k$, the region where the trivial solution becomes unstable is $r_{\text {th }}^{-}<$ $r<r_{\text {th }}^{+}$, where the pump limits are given by the neutral stability curves:

$$
\begin{aligned}
r_{\mathrm{th}}^{ \pm}= & \frac{-2 L \sigma\left(\Delta_{c}-a k^{2}\right)+p(1+\sigma)^{2}}{2 L^{2} \sigma} \\
& \pm \frac{(1+\sigma) \sqrt{-4 L p \sigma\left(\Delta_{c}-a k^{2}\right)-4 L^{2} \sigma^{2}+p^{2}(1+\sigma)^{2}}}{2 L^{2} \sigma} .
\end{aligned}
$$

Note that for negative cavity detunings the minimum of $r_{\text {th }}^{-}$occurs always at $k=0$, as in the absence of LFC [14].

Figure 2 shows the boundaries (3) of the instability region for $L=150$ and a negative detuning $\Delta_{c}=-1.5$, for which, according to Fig. 1(b), a bistable behavior is expected. Above the lower neutral stability curve $r_{\text {th }}^{-}$the trivial nonlasing solution destabilizes, choosing at threshold a wave number $k=0$, since the minimum of $r_{\mathrm{th}}^{-}$occurs at this wave number value. In the striped region above $r_{\text {th }}^{+}$, the off state becomes stable again (but now only versus perturbations with wave number included within such a region) and coexists with the solution that 


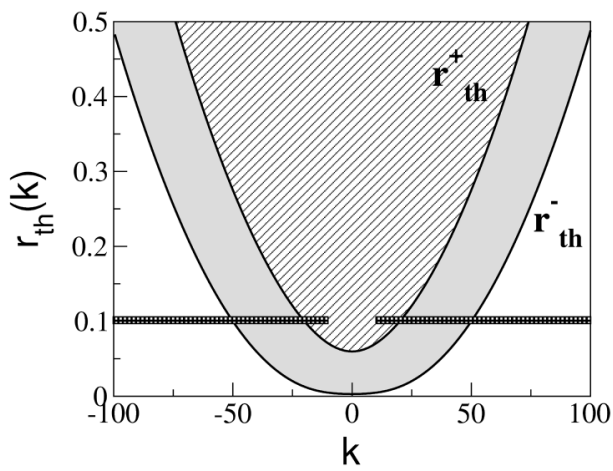

FIG. 2. Neutral stability curves for $p=250, \sigma=0.5, \Delta_{c}=$ $-1.5, a=0.01$, and $L=150$. In the white region only the nonlasing solution exists, in the gray region that solution is unstable versus perturbations of wave number $k$, and in the striped region the nonlasing and off-axis solutions coexist. The wide horizontal lines represent the Fourier filter to be discussed later in the text.

originated in $r_{\mathrm{th}}^{-}$, for which the wave number is still around 0 , since this is the wave number with highest gain.

It is well known [2] that the coexistence between two spatially homogeneous solutions can give rise to spatial solitons in optical cavities. In our case, however, the bistable striped region in Fig. 2 cannot be reached in practice, since at any given pump rate $r$ in the striped region, the nonlasing solution is destabilized by perturbations with transverse wave number $k$ within the gray region. In order to maintain stability of the zero solution, and hence the possibility of generating cavity solitons in this laser, one can prevent the growth of such large wave numbers by means of a low-pass spatial filter. This filter can be implemented in practice by placing a diaphragm in a far-field plane built inside the laser cavity [17]. Such a filter would keep the nonlasing solution stable, and bistability between this solution and the lasing state would allow the generation of cavity solitons. We have checked numerically that this is indeed what happens, as can be seen in Fig. 3(a). This figure displays (in solid line) the one-dimensional cross section of the intensity of a twodimensional cavity soliton generated by the model equations (1) in the presence of a sharp low-pass filter in Fourier space with cutoff wave number $|k|_{\max }=10$ (represented in Fig. 2 by a wide horizontal line at the pump rate used in the numerical simulations of Fig. 3). The filter has been implemented numerically via a sharp-edge step function, but more regular functions have been seen to lead to identical results, provided the filter is not too smooth to allow the growth of the unstable wave numbers within the gray region of Fig. 2. Simulations have been performed in a square lattice of $256 \times 256$ cells of size $\Delta x=0.05$, using a spectral split-step algorithm with integration time step $\Delta t=10^{-3}$. The intensity profile shows a clear localized structure in the center of the lattice. The width of the soliton, measured in terms of the full width at half maximum (FWHM), is in this case 0.39 spatial units, whose translation into physical units

083901-3

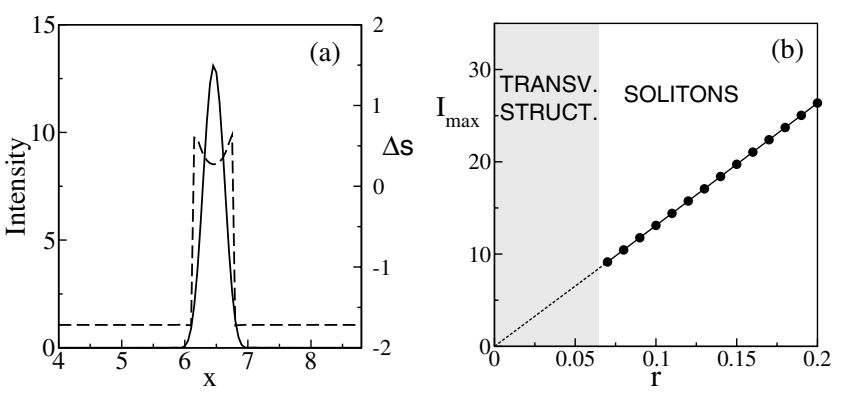

FIG. 3. (a) Horizontal cross section of a cavity soliton, in terms of the intensity (solid line, left $y$ axis) and the refraction index shift (dashed line, right $y$ axis). Parameters are those of Fig. 2, plus $b=0.5$ and $r=0.1$. The Fourier filter has a cutoff wave number $|k|_{\max }=10$. The seed is a 10-pixel-wide, sixthorder super-Gaussian placed at the center of the lattice. (b) Dependence of the peak intensity of the soliton on the pump rate $r$.

depends on the laser frequency and beam waist through the value of $a$ as defined above, since this quantity freely rescales the transverse dimensions of the model. The dashed line in Fig. 3(a) represents the quantity $\Delta s=$ $p \operatorname{Re}\left(\alpha \rho_{01}^{*}\right) /|\alpha|^{2}$, which is proportional to $n^{2}-1$ [15], where $n$ is the index of refraction of the medium. The abrupt increase in the index of refraction at the boundaries of the soliton indicates that the light localization is an index-guiding effect produced via self-focusing.

As mentioned earlier, in the presence of the Fourier filter described above the system exhibits bistability between the nonlasing solution and a homogeneous lasing state. The system chooses one state or the other depending on the initial conditions. The transverse spatial behavior shown in Fig. 3 is originated by seeding the soliton at the center of the lattice with a super-Gaussian initial condition of sufficient intensity. A unique characteristic of these cavity solitons with respect to similar structures in other systems is that their strength as well as the shape of their spatial profile can be modified by acting on the width and shape of the seeding signal, as well as on the profile of the spatial filter. We consider that this happens because of the interplay between diffraction, self-focusing (in particular, in the form of field-induced index guiding in the relatively dense gain medium), and spatial Fourier filtering. On the other hand, it is not related with any modulational instability [18] of the homogeneous lasing solution, since in our case (large negative detuning and close to threshold) such a solution is stable versus perturbations with nonzero wave number.

The dependence of the soliton characteristics with the laser parameters has been systematically investigated. In particular, the soliton peak intensity is plotted as a function of pumping in Fig. 3(b). In that figure, the shaded region corresponds to a pump range for which no bistability exists (cf. Fig. 2), accordingly no solitons are found numerically, and the laser exhibits a complex transverse dynamics (since pumping is well above the first lasing threshold, which is almost zero for this 

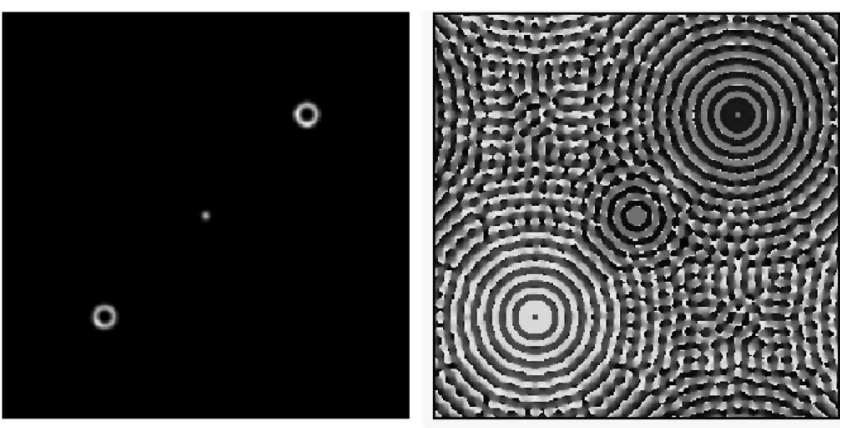

FIG. 4. Intensity (left) and phase (right) profiles of three coexisting localized structures. Parameters are $p=250, \sigma=$ $b=0.5, \Delta_{c}=-1.5, a=0.01, L=50, r=0.8$, and $|k|_{\max }=$ 14.

parameter's values; see again Fig. 2). Within the bistability region, the soliton maximum intensity is found to increase linearly with pumping, analogously to what happens in the standard case of Gaussian beams. Interestingly, this L-I line can be extrapolated to the first lasing threshold near zero [dashed line through the shaded area of Fig. 3(b)]. On the other hand, the full width at half maximum of the soliton is seen not to depend on $r$ (results not shown). The dependence of the soliton characteristics on other laser parameters is not so pronounced: in particular, the maximum intensity is basically independent of the local-field coefficient $L$, for instance (results not shown).

Localized structures with circular symmetry, not always including a central brightest spot, or even multihump structures, can also be generated and can coexist in the transverse plane. As an example, Fig. 4 shows the coexistence of a standard soliton with two doughnutlike localized structures. Such "plasticity" might be of interest for information processing, and, in particular, to control the interaction between neighboring cavity solitons or for the formation of bounded solitonic structures.

In conclusion, we have shown that, contrary to common belief, two-level lasers are able to generate and sustain cavity solitons, provided the amplifying medium is dense enough to make local-field effects important. These effects induce a bistability in the laser, which can be preserved in the presence of transverse diffraction by introducing a low-pass spatial Fourier filter within the cavity. Such filters (including filters based on spatial diffusion of saturated gain [19]) have already been used in the past to control the spatiotemporal dynamics of optical systems [20] and, in particular, of localized optical structures [19,21]. However, as far as we know, Fourier filtering had not been used so far to generate localized structures themselves, as reported here. This mechanism of soliton formation provides an alternative to more standard mechanisms such as those based in semiconductor or Kerr nonlinearities [4], but relies like them on the existence of a bistability in the system. On the other hand, it leads to solitons which are completely addressable and, in contrast to other cavity solitons known so far, their characteristics (peak intensity, size, and shape) can be controlled by the addressing beams and by the profile of the low-pass spatial Fourier filter. Multihump solitons, for instance, appear for wide enough initial injection beams.

We acknowledge financial support from MCyT (Spain) and FEDER (European Union), under Projects No. BFM2001-2159 and No. BFM2002-04369, and from the Catalan Government (Grants No. 2001-SGR00187 and No. 2001-SGR00223). V. A. acknowledges support from the CIRIT (Catalan Government) for a grant (No. 1998FI 00194PG).

[1] G. I. Stegeman and M. Segev, Science 286, 1518 (1999).

[2] Spatial Solitons, edited by W. Torruellas and S. Trillo (Springer, Berlin, 2001).

[3] N. N. Rosanov and G.V. Khodova, J. Opt. Soc. Am. B 7, 1065 (1990); M. Tlidi et al., Phys. Rev. Lett. 73, 640 (1994); M. Brambilla et al., Phys. Rev. Lett. 79, 2042 (1997); D. Michaelis et al., Phys. Rev. A 56, R3366 (1997); V. J. Sánchez-Morcillo et al., Opt. Lett. 25, 957 (2000).

[4] M. Kreuzer et al., Mol. Cryst. Liq. Cryst. 207, 219 (1991); M. Saffman et al., Opt. Lett. 19, 518 (1994); V. B. Taranenko et al., Phys. Rev. Lett. 81, 2236 (1998); B. Schäpers et al., Phys. Rev. Lett. 85, 748 (2000); S. Barland et al., Nature (London) 419, 699 (2002).

[5] V. Y. Bazhenov et al., Proc. SPIE Int. Soc. Opt. Eng. 1840, 183 (1992); N. N. Rosanov et al., Physica (Amsterdam) 96D, 272 (1996); V. B. Taranenko et al., Phys. Rev. A 56, 1582 (1997).

[6] R. Vilaseca et al., Phys. Rev. Lett. 87, 083902 (2001).

[7] D. Bedeaux and N. Bloembergen, Physica (Amsterdam) 69, 57 (1973).

[8] R. Friedberg et al., Phys. Rev. A 40, 2446 (1989); 42, 494 (1990).

[9] F. A. Hopf et al., Phys. Rev. A 29, 2591 (1984).

[10] J. J. Maki et al., Phys. Rev. Lett. 67, 972 (1991).

[11] M. P. Hehlen et al., Phys. Rev. Lett. 73, 1103 (1994); C. M. Bowden, Phys. World 7, 24 (1994).

[12] F. Sanchez et al., Phys. Rev. A 61, 033817 (2000); M. Fromager et al., Phys. Rev. A 61, 053804 (2000).

[13] R. Vilaseca and C. Weiss, Dynamics of Lasers (VCH, Weinheim, 1991).

[14] P. K. Jakobsen et al., Phys. Rev. A 49, 4189 (1994).

[15] V. Ahufinger, Ph.D. thesis, Universitat Autònoma de Barcelona, 2002.

[16] O. G. Calderón et al., Phys. Rev. A 67, 043812 (2003).

[17] C. O. Weiss et al., Appl. Phys. B 68, 151 (1999).

[18] K. Staliunas and V. J. Sánchez-Morcillo, Opt. Commun. 139, 306 (1997).

[19] K. Staliunas, Phys. Rev. A 61, 053813 (2000).

[20] R. Martin et al., Phys. Rev. Lett. 77, 4007 (1996); A.V. Mamaev and M. Saffman, Phys. Rev. Lett. 80, 3499 (1998); E. Benkler et al., Phys. Rev. Lett. 84, 879 (2000).

[21] P. L. Ramazza et al., Phys. Rev. E 65, 066204 (2002). 